\title{
Neuronal connectivity inference from spike trains using an empirical probabilistic causality measure Paulo Aguiar*1,2 and Miguel Rodrigues ${ }^{3}$
}

\author{
Address: ${ }^{1}$ Centro de Matemática da Universidade do Porto, 4169-007 Porto, Portugal, 2 Instituto de Biologia Molecular e Celular, 4150-180 Porto, \\ Portugal and ${ }^{3}$ Computer Science Dept., University of Porto, 4169-007 Porto, Portugal \\ Email: Paulo Aguiar* - pauloaguiar@fc.up.pt \\ * Corresponding author
}

from Eighteenth Annual Computational Neuroscience Meeting: CNS*2009

Berlin, Germany. 18-23 July 2009

Published: 13 July 2009

BMC Neuroscience 2009, I0(Suppl I):PI69 doi:I0.II86/I47|-2202-I0-SI-PI69

This abstract is available from: http://www.biomedcentral.com/I47I-2202/I0/SI/PI69

(c) 2009 Aguiar and Rodrigues; licensee BioMed Central Ltd.

To fully understand the computational properties of a neural network, it is crucial to know the network's connectivity. Unfortunately, connectivity inference using easily accessible data such as extracellular spike recordings is a difficult task. The difficulty arises mainly from problems related with causality, polysynaptic (indirect) connectivity, inhibition and variable time delays, just to name a few. Different methods have been proposed for connectivity inference using, for example, mutual information $[1,2]$, partial directed coherence [3], direct neuronal dynamics parameters fitting [4] or parameter estimation using Bayesian approaches [5]. However, we argue that most of these methods are too complex to implement, hard to apply to spike times series or simply do not provide appropriate estimations using benchmark synthetic data.

Here we present an alternative method that takes directly the series of spike times from multiple sources and produces a probabilistic connectivity matrix, wherein each entry provides a confidence level for the existence of a connection (excitatory or inhibitory) for a particular source/target pair. Instead of using measures which are directly or indirectly related to correlations, our method introduces an empirical measure of causality that works on top of spike time probability distributions obtained from the spike times series. This approach allows us to cope with variable time delays, causality, inhibitory connections and provides an estimate for the degree of connection (monosynaptic, disynaptic, etc.). Our method includes heuristics but only one parameter needs to be set: the causality time window. This value defines the maximum time delay between two events that are said to be related and is used to constrain the causality search space.

Our method has been tested using synthetic data from simulations where the neuronal connectivity is known $a$ priori. Two types of neuron models were tested in different network simulations: single-compartment Hodgkin-Huxley model with static synapses and Poisson spiking units modulated by stochastic synapses.

\section{Acknowledgements}

The first author was supported by the Centro de Matemática da Universidade do Porto, financed by FCT through the programs POCTI and POSI, with Portuguese and European Community structural funds.

\section{References}

I. Shiono S, Yamada S, Nakashima M, Matsumoto K: Information theoretic analysis of connection structure from spike trains. In Advances in Neural information Processing Systems 51992 Edited by: Hanson SJ, Cowan JD, Giles CL. Morgan Kaufmann Publishers, San Francisco, CA; 1993:51 5-522.

2. Bettencourt LM, Stephens G], Ham MI, Gross GW: The functional structure of cortical neuronal networks grown in vitro. Phys Rev E 2007, 75:021915.

3. Takahashi DY, Baccalá LA, Sameshima K: Connectivity inference between neural structures via partial directed coherence. Journal of Applied Statistics 2007, 34: I259-I273.

4. Makarov VA, Panetsos F, De Feo O: A method for determining neural connectivity and inferring the underlying network dynamics using extracellular spike recordings. J Neurosci Methods 2005, 1 44:265-279.

5. Rajaram S: Poisson networks: a model for structured point processes. Al and Statistics 2005, I 0:. 